\title{
5 Research Square

\section{Impact of obstructive sleep apnea on health-related quality of life in patients with partial laryngectomy for laryngeal cancer}

\section{Min Chen}

Fudan University Eye Ear Nose and Throat Hospital

\section{Fang Shi}

Fudan University Eye Ear Nose and Throat Hospital

\section{Haitao Wu}

Fudan University Eye Ear Nose and Throat Hospital

\section{Lei Cheng}

Fudan University Eye Ear Nose and Throat Hospital

\section{Peijie He}

Fudan University Eye Ear Nose and Throat Hospital

\section{Qian Jin}

Fudan University Eye Ear Nose and Throat Hospital

Jing jing Huang ( $\nabla$ gennie_xuan@163.com )

Fudan University Eye Ear Nose and Throat Hospital https://orcid.org/0000-0002-9237-6595

\section{Research Article}

Keywords: Obstructive sleep apnea, laryngeal cancer, health-related quality of life, risk factor

Posted Date: January 18th, 2022

DOI: https://doi.org/10.21203/rs.3.rs-1192063/v1

License: (9) This work is licensed under a Creative Commons Attribution 4.0 International License. Read Full License 


\section{Abstract \\ Purpose}

To evaluate the possible risk factors of obstructive sleep apnea (OSA) and its impact on the healthrelated quality of life (HRQoL) in patients with partial laryngectomy for laryngeal cancer.

\section{Methods}

Patients who underwent partial laryngectomy for laryngeal cancer completed overnight polysomnography (PSG) home sleep tests and quality of life questionnaires. The MOS 36-item ShortForm Health Survey (SF-36) questionnaire was used to investigate factors influencing HRQoL.

\section{Results}

Fifty-nine patients completed PSG tests and quality of life questionnaires, with $74.6 \%$ demonstrating evidence of OSA (Apnea-hyponea Index (AHI) ranging 5.2-49.8 per hour, median 12.8 per hour). There were significant differences in tumor area and neck dissection between OSA group and non-OSA groups. Patients with OSA had significant lower scores of body pain, general health, and health transition in SF36 domains. Independent factors associated with general health were identified as tobacco use (OR=4.716, 95\% Cl: 0.207-2.894), alcohol use (OR=3.193, 95\% Cl: 0.042-2.28), and OSA (OR=11.336, 95\% Cl: 0.994-3.862).

\section{Conclusion}

Larger tumor area and neck dissection might be associated with an increased risk for developing OSA in patients with partial laryngectomy for laryngeal cancer. OSA partially mediated the effect on physical health, including body pain, general health, and health transition. It is important to be aware of the potential impact of OSA on diminished HRQoL of these patients.

\section{Introduction}

More than 180,000 new cases worldwide were diagnosed with laryngeal cancer in 2020[1]. Previously, laryngeal cancer management was concentrated primarily on tumor control and survival rate but has evolved to incorporate functional and health-related quality of life (HRQoL) outcomes[2]. Within patients who present with laryngeal cancer managed with surgery, radiation therapy (RT), chemotherapy, or a combination of these modalities, the diminished HRQoL have been investigated to varied extents[3]. Moreover, lesser studies have focused on its influencing factors. Two major problems, swallowing disorders and dysphonia, have been shown to influence HRQoL negatively[4]. Previous researches indicated that patients with laryngeal cancer might need support regarding swallowing and voice 
rehabilitation[5]. Thus, exploration of related-factors is favorable to optimize the treatment regimens, improve the therapeutic outcomes, and eventually better HRQoL of these patients.

Fatigue and sleep disturbance have been increasingly recognized as health issues that negatively affect HRQoL for patients with other cancer[6]. Existing studies proposed that fatigue and sleep disturbance also persisted among patients with laryngeal cancer after treatment[7]. Up to now, far too little attention has been paid to identifying the association between sleep with HRQoL in laryngeal cancer patients. An often overlooked cause of sleep disorders in this population was obstructive sleep apnea (OSA). Previous studies reported incidence of OSA afterward therapy was extensive, ranging from $12 \%-96 \%[8]$. And significant risk factors for OSA were defined in recent studies[9, 10]. However, the association between tumor size, clinical stage, surgical method, radiotherapy and OSA is still inconclusive. Furthermore, the severity of OSA and nocturnal hypoxemia was independently associated with all-cancer risk based on biological models[11]. Clinicians should recognize the prevalence, aetiology, and correct management of OSA after therapy for laryngeal cancer[12].

HRQoL assessment requires an instrument that is multi-dimensional, standardized, easily understandable, and workable. The MOS 36-item Short-Form Health Survey (SF-36) which has physical and mental scales measuring nine domains of HRQoL was valid, credible, and compatible in the Chinese population[13]. A variety of specific instruments may assess different aspects of HRQoL in patients with laryngeal cancer, including M.D. Anderson Dysphagia Inventory (MDADI), voice-related quality of life (VRQOL), and Hospital anxiety and depression scale (HADS)[14]. The SF-36 was confirmed to have the best validity, reliability, and responsiveness for general health-status measures of patients with OSA[15].

It might be concluded that convincing evidence for the association between OSA with HRQoL in patients treated for laryngeal cancer was lacking. Hence, the main purpose of this research was to evaluate the impact of OSA on HRQoL of patients with partial laryngectomy. Additionally, prevalence and possible risk factors for OSA in these patients were investigated.

\section{Methods}

From April 2018 to March 2020, patients with laryngeal squamous cell carcinoma (LSCC) underwent partial laryngectomy in Eye, Ear, Nose, and Throat Hospital, Fudan University were recruited postoperatively. The study was approved by the Ethics Committee and the Institution Review Board of the Eye, Ear, Nose, and Throat Hospital, Fudan University, Shanghai, China (Ethical approval NO. 2020014-1). Written informed consent was obtained from each patient. Inclusion criteria were a clear diagnosis of LSCC, no dyspnea during continuous tube-plugging for at least a month, planned tracheostomy extubation, no recurrence of the tumor, no medical history of other malignancies, ability to undergo the polysomnography (PSG), and adequate knowledge of Chinese.

Demographic and clinical characteristics were respectively collected via chart review and self-reported. Body mass index (BMI) was calibrated as patient's weight in kilograms divided by the square of height in meters. Tobacco use was defined as a current or history of daily tobacco consumption. Alcohol use was 
defined as a daily intake of more than $80 \mathrm{~mL}$ of pure alcohol per day. Smoking index was calculated as the number of cigarettes consumed per day multiply by years of smoking. Drinking index was calculated as the volume of pure alcohol consumed per day multiply by years of drinking. The clinical staging was determined using Tumor, Node, Metastasis (TNM) staging system outlined in the American Joint Committee on Cancer's AJCC Staging Manual, 8th edition. Tumor area (length * width) and tumor depth were manually extracted from pathology reports. Surgical treatment included partial laryngectomy with or without neck dissection. Surgical method included Supracricoid partial laryngectomy (SCPL) and Vertical partial laryngectomy (VPL).

\section{PSG}

All participants underwent an overnight PSG home sleep test (Embletta MPR) in their own house at the night before extubation. The monitor consisted of nasal airflow, oxygen saturation, and electrocardiograph. An experienced sleep laboratory technician scored all tests based on standard criteria. Individuals with obstructive apnea-hypopnea index $(\mathrm{AHI}) \geq 5$ per hour were classified as OSA

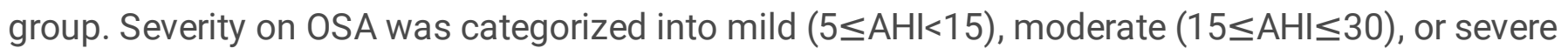
$(\mathrm{AHI}>30)$.

\section{HR-QoL assessment}

SF-36 was applied to assess overall HR-QoL at the same time as PSG test[16]. This questionnaire contains 36 items that describe nine dimensions of health: physical function (PF), role physical function $(\mathrm{RF})$, body pain (BP), general health (GH), vitality (VT), social function (SF), role emotion (RE), mental health $(\mathrm{MH})$, and health transition $(\mathrm{HT})$ [17]. It presents a final score ranging from 0 to 100 , in which 0 corresponds to the worst health condition and 100 corresponds to the best health status.

\section{Statistical analysis}

Data were analysed using SPSS version 20.0 (IBM Corporation, Chicago, Illinois, USA) and R software (version 3.6.1; http://www.R-project.org) with the ggplot2, magrittr, checkmate, forestplot, and ggpubr libraries added. The $P$ values $<0.05$ were considered statistically significant. Absolute numbers and percentages were identified for categorial variables. Mean and SD were calculated for continuous variables. Continuous variables were converted to binary variables and ordered categorical variables according to the median numbers and quartiles, respectively. Categorical variables were compared using Chi-squared test or Fisher's exact tests, depending on the number of observations. Ordinal categorical variables were compared using Mantel-Haenszel Chi-squared test. Continuous variables were analyzed using Student's t test or Mann-Whitney U test, as appropriate for the type of distribution. Principal component analysis (PCA) combines K-means clustering to identify 2 clusters based on PSG-related parameters. The comparisons of HR-QoL were visualized using radar charts. Multivariate analysis by Ordinal regression model was shown via the forest plot.

\section{Results}


A total of 78 patients who underwent partial laryngectomy for laryngeal cancer were eligible for this study, of which $75.6 \%$ responded by answering the questionnaire. Finally, 59 patients were included in the analysis, of whom $96 \%$ were male and $4 \%$ were female, with a mean age of $60.1 \pm 8.53$ years (range from 33 to 80). Fifteen patients received neck dissection, 12 patients received adjuvant radiation, and 2 patients received chemoradiotherapy. Based on PSG, forty-four patients $(74.6 \%)$ were diagnosed with OSA (AHI range from 5.2 to 49.8 per hour, median 12.8 per hour), specifically 25 (42.4\%) with mild OSA, $11(18.6 \%)$ with moderate OSA, and $8(13.6 \%)$ with severe OSA. Baseline characteristics of the cohort were summarized in Table 1. 
Table 1

Demographic and clinical characteristics of 59 patients

\begin{tabular}{|c|c|c|c|}
\hline Characteristics & No-OSA $(n=15)$ & OSA $(n=44)$ & $P$ \\
\hline Age (years) & $57.2 \pm 10.65$ & $61.05 \pm 7.57$ & $0.133^{\mathrm{a}}$ \\
\hline \multicolumn{4}{|l|}{ Gender } \\
\hline Male & $15(100 \%)$ & $42(95.5 \%)$ & $0.401^{\mathrm{c}}$ \\
\hline Female & 0 & $2(4.5 \%)$ & \\
\hline BMI & $22.4 \pm 2.11$ & $23.05 \pm 3.08$ & $0.45^{\mathrm{a}}$ \\
\hline \multicolumn{4}{|l|}{ Tobacco use } \\
\hline Current/previous & $10(66.7 \%)$ & 35 (79.5\%) & $0.311^{\mathrm{b}}$ \\
\hline Never & $5(33.3 \%)$ & $9(20.5 \%)$ & \\
\hline Smoking index & $433.33 \pm 416.91$ & $600.91 \pm 456.64$ & $0.215^{a}$ \\
\hline \multicolumn{4}{|l|}{ Alcohol use } \\
\hline Current/previous & $5(33.3 \%)$ & $15(34.1 \%)$ & $0.957^{b}$ \\
\hline Never & $10(66.7 \%)$ & $29(65.9 \%)$ & \\
\hline Drinking index & $800 \pm 1207.12$ & $959.09 \pm 1558.52$ & $0.721^{a}$ \\
\hline \multicolumn{4}{|l|}{ Hypertension } \\
\hline Yes & $3(20 \%)$ & $18(40.9 \%)$ & $0.144^{b}$ \\
\hline No & $12(80 \%)$ & $26(59.1 \%)$ & \\
\hline \multicolumn{4}{|l|}{ Diabetes } \\
\hline Yes & $1(6.7 \%)$ & $7(15.9 \%)$ & $0.367^{b}$ \\
\hline No & $14(93.3 \%)$ & 37 (84.1\%) & \\
\hline \multicolumn{4}{|l|}{ T stages } \\
\hline $\mathrm{T} 1$ & $4(26.7 \%)$ & $19(43.2 \%)$ & $0.482^{d}$ \\
\hline T2 & $7(46.7 \%)$ & $16(36.4 \%)$ & \\
\hline T3 & $4(26.7 \%)$ & $7(15.9 \%)$ & \\
\hline $\mathrm{T} 4$ & 0 & $2(4.5 \%)$ & \\
\hline
\end{tabular}

a Student's t test; ${ }^{b}$ Chi-squared test; ${ }^{c}$ Fisher's exact test; ${ }^{d}$ Mantel-Haenszel Chi-squared test. 


\begin{tabular}{|c|c|c|c|}
\hline Characteristics & No-OSA $(n=15)$ & OSA $(n=44)$ & $P$ \\
\hline \multicolumn{4}{|l|}{$\mathrm{N}$ stages } \\
\hline No & $15(100 \%)$ & $34(77.3 \%)$ & $0.128^{d}$ \\
\hline $\mathrm{N} 1$ & 0 & $6(13.6 \%)$ & \\
\hline N2 & 0 & $4(9.1 \%)$ & \\
\hline \multicolumn{4}{|l|}{ Clinical stages } \\
\hline I & $4(26.7 \%)$ & $16(36.4 \%)$ & $0.855^{d}$ \\
\hline II & $7(46.7 \%)$ & $12(27.3 \%)$ & \\
\hline III & $4(26.7 \%)$ & $11(25.0 \%)$ & \\
\hline IV & 0 & $5(11.4 \%)$ & \\
\hline Tumor area & $1.5793 \pm 0.55$ & $2.9475 \pm 3.39$ & $0.013^{a}$ \\
\hline Tumor depth & $0.76 \pm 0.28$ & $0.95 \pm 1.11$ & $0.516^{a}$ \\
\hline \multicolumn{4}{|l|}{ Surgical method } \\
\hline Supracricoid partial laryngectomy & $6(40 \%)$ & $16(36.4 \%)$ & $0.801^{\mathrm{b}}$ \\
\hline Vertical partial laryngectomy & $9(60 \%)$ & $28(63.6 \%)$ & \\
\hline Neck dissection & & & $0.007^{c}$ \\
\hline Yes & 0 & $15(34.1 \%)$ & \\
\hline No & $15(100 \%)$ & $29(65.9 \%)$ & \\
\hline \multicolumn{4}{|l|}{ Radiation therapy } \\
\hline Yes & $3(20 \%)$ & $9(20.5 \%)$ & $0.97^{b}$ \\
\hline No & $12(80 \%)$ & 35 (79.5\%) & \\
\hline \multicolumn{4}{|l|}{ Chemoradiotherapy } \\
\hline Yes & $1(6.7 \%)$ & $1(2.3 \%)$ & $0.417^{\mathrm{b}}$ \\
\hline No & $14(93.3 \%)$ & $43(97.7 \%)$ & \\
\hline
\end{tabular}

The tumor area in OSA group was significantly larger than that in non-OSA group $(2.95 \pm 3.39 \mathrm{vs}$. $1.58 \pm 0.55, P=0.013)$. In the OSA group there were significantly more patients underwent neck dissection than these in the non-OSA group ( $34.1 \%$ vs. $0 \%, P=0.007)$. However, both groups were similar in the 
distribution of age, gender, BMI, tobacco use, alcohol use, clinical stage, tumor depth, surgical method, history of hypertension, diabetes, radiation, and chemoradiotherapy.

A radar-chart presents an overview of the all SF-36 domains between non-OSA group and OSA subgroups (Fig. 1A). Patients with partial laryngectomy had lower scores of RF, GH, RE, and HT than the scores of PF, $\mathrm{BP}, \mathrm{VT}, \mathrm{SF}$, and MH. Severe-OSA subgroup had the lowest scores in majority of domains compared with that of other three subgroups.

In order to study the impact of OSA on the quality of life after partial laryngectomy, patients were clustered based on PSG-related parameters showed in Table 2. Dimensionality-reduction of PSG-related parameters were performed using PCA (Supplementary Material). Combined with K-means clustering, cluster 1 and cluster 2 were identified (Fig. 1B). The first component, labelled "respiratory events", had the greatest loadings on oxygen desaturation index (OD) and AHI; the second component, labelled "oxygen saturation", had the greatest loading on mean Pulse Oximeter Oxygen Saturation $\left(\mathrm{SpO}_{2}\right)$.

Correspondingly, each mean score of cluster 1 was higher than that of cluster 2 in the nine dimensions (Fig. 1C). Further statistical analysis was shown as a violin plot (Fig. 1D). It revealed 9 domains exhibited different distribution and significantly different levels of $\mathrm{BP}, \mathrm{GH}$, and $\mathrm{HT}$ between two clusters. Although it showed the phenotype of worse condition in PF, RF, VT, SF, RE, and MH of cluster 2, no statistically significant differences in these levels were observed. 
Table 2

PSG-related parameters of 59 patients

\section{Number of patients (\%)}

$\mathrm{AHI}$

$<5$

$5-15$

15-30

$>30$

SAHI

OD

Longest Apnea Time

Minimum $\mathrm{SpO}_{2}$

$>90 \%$

$85-90 \%$

$80 \%-85 \%$

$<80 \%$

Mean $\mathrm{SpO}_{2}$

Time in $\mathrm{SpO}_{2}<90 \%$

Abbreviation: AHI, Apnea-hyponea Index. SAHI, Supine apnea-hypopnea index. OD, oxygen desaturation index. $\mathrm{SpO}_{2}$, Pulse Oximeter Oxygen Saturation.
$15(25.4 \%)$

$25(42.4 \%)$

$11(18.6)$

$8(13.6 \%)$

$19.28 \pm 16.33$

$11.36 \pm 11.08$

$54.22 \pm 29.24$

$20(33.9 \%)$

$25(42.4 \%)$

$11(18.6 \%)$

$3(5.1 \%)$

$95.76 \pm 1.38$

$0.837 \pm 1.846$

Other factors, tobacco use, alcohol use, tumor area, and neck dissection, were screened to be also significantly associated with specific health domains (BP, GH, and $\mathrm{HT}$ ) by univariate analysis. Multivariate analysis by Ordinal regression model was shown in the forest plot (Fig. 2). No factor was significantly associated with BP in the multivariate model (Fig. 2A). Further independent factors associated with $\mathrm{GH}$ were tobacco use (OR=4.716, 95\% Cl: $0.207-2.894)$, alcohol use (OR=3.193, 95\% Cl: 0.042-2.28), and OSA (OR=11.336, 95\% Cl: 0.994-3.862) (Fig. 2B). Tobacco use was the only significant factor that influenced HT independently with an HR of 4.702 and $95 \% \mathrm{Cl}$ of. $0.192-2.903$ (Fig. 2C).

\section{Discussion}

Treatment of laryngeal cancers modify the upper airway anatomy and thereby increase the risk of OSA[8]. In this study, there was a positive screening for OSA in $74.6 \%$ of laryngeal cancer patients underwent partial laryngectomy, which was significantly higher than the reported prevalence of OSA in the general 
population (15\%)[18]. Similar findings have also been demonstrated by others[9, 19]. However, the incidence of OSA in our study was lower than that of those studies ( $82.5 \%$ and $92.3 \%$, respectively). This discrepancy was most likely due to treatment bias as clinical stages and treatment modalities were diverse. All patients in our study received partial laryngectomy, $25 \%$ of them received neck dissection or adjuvant radiation or chemoradiotherapy. This proportion might be higher in previous studies, while were not elucidated. Another possible explanation for this was the different screening tools used for OSA. Nevertheless, these researches highlighted that treatment increase risk of developing OSA.

We compared clinical characteristics of posttreatment patients grouped by OSA status and found evidence of significant associations, which were mentioned in only a minority of studies. Firstly, larger tumor area might be an important predictor for developing OSA, this observation was consistent with findings from others[20]. The larger tumor area indicated the greater structural alterations of upper airway which was regarded as the basis of OSA. Additionally, we have highlighted for the first time that neck dissection was a significant risk factor for OSA. The mechanisms involved remain unknown and require further investigation. We speculated that damage to muscles and nerves and decrease of inspiratory strength might be responsible for the OSA after neck dissection[21]. Relatively more researches focused on the impact of RT on OSA provided inconsistent conclusions. Chronic edema after RT would favor OSA was reported by some literature[22]. Our findings pointed to no significant association between RT and OSA risk in laryngeal cancers which in line with a recent systematic review[23]. Irradiation would hypothetically stiffen the soft tissues and subsequently would not favor OSA. Negative findings also appeared in other clinical characteristics (Table 1). Above all, screening of OSA is highly recommended in laryngeal cancer patients after treatment, especially for those with larger tumor area and neck dissection.

The current study revealed OSA might contribute to impair HR-QoL among laryngeal cancer patients after treatment. Worse status in severe OSA group was observed in majority of the measured domains. Despite recent awareness of the association between OSA and decreased quality of life in laryngeal cancer patients, for the first time, we have provided evidence for this via using the validated questionnaire SF-36. To obtain a complete understanding of effects of OSA on HR-QoL, it is essential to involve more PSGrelated parameters in analysis not limited to $\mathrm{AHI}$. In this study, two clusters next redefined using PCA followed by K-means clustering have different characteristics. In general, cluster 2 had lower mean score of each dimension than cluster 1 in variable degrees (Fig. 1C). Particularly, OSA-related condition significantly correlated to the BP, GH, and HT domains of SF-36, suggesting that patients with OSA indicated a higher likelihood of exhibiting physical limitations (Fig. 1D). This raises the question whether or not OSA influences patient's health in these 3 domains independently. Since we found an odd ratio of 11 between two clusters in $\mathrm{GH}$, OSA was most probably had significant $\mathrm{GH}$ impairments postoperatively in patients with laryngeal cancer. A possible explanation for these might be that OSA in patients with partial laryngectomy involves intermittent hypoxia. As is well known, hypoxia diminished physiological functions and the overall quality of life. Previous studies have shown continuous positive airway pressure (CPAP) may help to improve HR-QoL, especially physical performance[24, 25]. However, CPAP therapy for patients with partial laryngectomy was not adequate. Recently, a case report indicated that subjective symptoms of sleepiness would be improved when severe OSA was controlled by CPAP after concurrent 
chemoradiotherapy for laryngeal cancer[26]. A further study with more focus on impacts of CPAP treatment on the HR-QoL in patients with partial laryngectomy is therefore suggested. This concerns whether CPAP is recommended for patients with OSA after treatment for laryngeal cancer.

Our interpretation of correlations between different clinical characteristics and these 3 domains was presented on the forest plot based on Multivariate analysis (Fig. 2). The findings confirm previous reports in general population of poor HR-QoL in smokers and drinkers[27]. The effect of smoking and drinking, the well-known risk factors of laryngeal cancer, on quality of life in postoperative patients was not fully understood. In the postoperative GH score evaluation, there was a statistically significant difference in favor of physical function limitations due to tobacco and alcohol use (Fig. 2B). Additionally, tobacco use was the only risk factor independently related to HT (Fig. 2C). Numerous investigations have already proposed smoking and drinking were risk factors affecting the recurrence and mortality of patients with laryngeal cancer[28]. According to our data, their role in quality of life impairments should be underlined. All patients with known laryngeal cancer would be strongly encouraged to quit smoking and drinking.

Another concern is lack of the criteria for tracheostomy extubation in patients with partial laryngectomy to data. All patients in this study met the inclusion criterion that no dyspnea during continuous tubeplugging for at least a month, however $74.6 \%$ of these were diagnosed with OSA based on overnight PSG tests. These findings suggested that some patients who planned tracheostomy extubation still had nocturnal hypoxemia. Additionally, considering the negative impact of OSA on quality of life in patients with partial laryngectomy for laryngeal cancer, further study was recommended to investigate whether PSG would be a useful tool in the decision-making about tracheostomy extubation.

Several limitations should be considered. PSG was not performed before surgery and the OSA status in baseline was not evaluated. It should be pointed that HR-QoL assessment is inherently subjective although SF-36 was proved to be valid and reliable in the Chinese population. Further work is required to evaluate the HR-QoL of patients after treatment for OSA (CPAP therapy) so that the impact of OSA on HRQoL would be further confirmed.

\section{Conclusion}

Larger tumor area and neck dissection might be associated with an increased risk for developing OSA in patients with partial laryngectomy for laryngeal cancer. OSA partially mediated the effect on physical health, including $\mathrm{BP}, \mathrm{GH}$, and $\mathrm{HT}$. It is important to be aware of the potential impact of OSA on diminished HRQoL of these patients.

\section{Declarations}

Funding: This work was supported by Science and Technology Commission of Shanghai Municipality of China (Grant numbers 20Y11901900 and 21Y11912000) and the Health and Family Planning Commission of Shanghai Municipality of China (Grant number 2019SY059). 
Competing Interests: The authors have no relevant financial or non-financial interests to disclose.

Availability of data and material: Not applicable.

Code availability: Not applicable.

Author contributions: Min Chen, Fang Shi, and Jingjing Huang contributed to the study conception and design. Material preparation, data collection and analysis were performed by Min Chen, Fang Shi and Qian Jin. The first draft of the manuscript was written by Min Chen. Haitao Wu, Lei Cheng, Peijie He, Jingjing Huang reviewed and edited the manuscript. Jingjing Huang corrected the manuscript. All authors read and approved the final manuscript.

Ethics approval: This study was performed in line with the principles of the Declaration of Helsinki.

Approval was granted by the ethics committee of the Eye, Ear, Nose, and Throat Hospital, Fudan University, Shanghai, China (NO. 2020014-1).

Consent to participate: Informed consent was obtained from all individual participants included in the study.

Consent for publication: Not applicable.

\section{References}

1. Sung H, Ferlay J, Siegel R L, Laversanne M, Soerjomataram I, Jemal A, Bray F (2021) Global Cancer Statistics 2020: GLOBOCAN Estimates of Incidence and Mortality Worldwide for 36 Cancers in 185 Countries. CA Cancer J Clin 71(3):209-249. https://doi.org/10.3322/caac.21660

2. Kolator M, Kolator P, Zatonski T (2018) Assessment of quality of life in patients with laryngeal cancer: A review of articles. Adv Clin Exp Med 27(5):711-715. https://doi.org/10.17219/acem/69693

3. O'Hara J, Whitmarsh A, Pring M, Thomas S, Ness A (2021) Quality of life following treatment for T1a glottic cancer with surgery or radiotherapy: outcomes from the Head and Neck 5000 cohort. Clin Otolaryngol. https://doi.org/10.1111/coa.13849

4. Tuomi L, Karlsson T, Johansson M, Finizia C (2015) Health-related quality of life and voice following radiotherapy for laryngeal cancer--a comparison between glottic and supraglottic tumours. Acta Oncol 54(1):73-79. https://doi.org/10.3109/0284186X.2014.925576

5. Tuomi L, Karlsson T (2020) Voice Quality, Function, and Quality of Life for Laryngeal Cancer: A Prospective Longitudinal Study Up to 24 Months Following Radiotherapy. Ear Nose Throat J 100(10_suppl):913S-920S. https://doi.org/10.1177/0145561320929941

6. Joly F, Ahmed-Lecheheb D, Kalbacher E, Heutte N, Clarisse B, Grellard J M, Gernier F, Berton-Rigaud D, Tredan O, Fabbro M, Savoye A M, Kurtz J E, Alexandre J, Follana P, Delecroix V, Dohollou N, RoemerBecuwe C, De Rauglaudre G, Lortholary A, Prulhiere K, Lesoin A, Zannetti A, N'Guyen S, Trager-Maury S, Chauvenet L, Abadie Lacourtoisie S, Gompel A, Lhomme C, Floquet A, Pautier P (2019) Long-term 
fatigue and quality of life among epithelial ovarian cancer survivors: a GINECO case/control VIVROVAIRE I study. Ann Oncol 30(5):845-852. https://doi.org/10.1093/annonc/mdz074

7. Hammermuller C, Hinz A, Dietz A, Wichmann G, Pirlich M, Berger T, Zimmermann K, Neumuth T, Mehnert-Theuerkauf A, Wiegand S, Zebralla V (2021) Depression, anxiety, fatigue, and quality of life in a large sample of patients suffering from head and neck cancer in comparison with the general population. Bmc Cancer 21(1):94. https://doi.org/10.1186/s12885-020-07773-6

8. Gavidia R, Dunietz G L, O'Brien L, Shannon C, Schuetz S, Spector M, Swiecicki P, Chervin R D (2021) Obstructive sleep apnea in patients with head and neck cancer: a systematic review. J Clin Sleep Med 17(5):1109-1116. https://doi.org/10.5664/jcsm.9134

9. Ouyang L, Yi L, Wang L, Tang Q, Yang X, Li S (2019) Obstructive sleep apnea in patients with laryngeal cancer after supracricoid or vertical partial laryngectomy. J Otolaryngol Head Neck Surg 48(1):26. https://doi.org/10.1186/s40463-019-0347-6

10. Huppertz T, Horstmann V, Scharnow C, Ruckes C, Bahr K, Matthias C, Gouveris H (2021) OSA in patients with head and neck cancer is associated with cancer size and oncologic outcome. Eur Arch Otorhinolaryngol 278(7):2485-2491. https://doi.org/10.1007/s00405-020-06355-3

11. Tan B K J, Teo Y H, Tan N K W, Yap D W T, Sundar R, Lee C H, See A, Toh S T (2021) Association of obstructive sleep apnea and nocturnal hypoxemia with all-cancer incidence and mortality: a systematic review and meta-analysis. J Clin Sleep Med. https://doi.org/10.5664/jcsm.9772

12. Ralli M, Campo F, Angeletti D, Allegra E, Minni A, Polimeni A, Greco A, de Vincentiis M (2020) Obstructive Sleep Apnoea in Patients Treated for Head and Neck Cancer: A Systematic Review of the Literature. Medicina (Kaunas) 56(8):399. https://doi.org/10.3390/medicina56080399

13. Lam C L, Tse E Y, Gandek B, Fong D Y (2005) The SF-36 summary scales were valid, reliable, and equivalent in a Chinese population. J Clin Epidemiol 58(8):815-822. https://doi.org/10.1016/j.jclinepi.2004.12.008

14. Wulff N B, Dalton S O, Wessel I, Arenaz Bua B, Lofhede H, Hammerlid E, Kjaer T K, Godballe C, Kjaergaard T, Homoe P (2021) Health-Related Quality of Life, Dysphagia, Voice Problems, Depression, and Anxiety After Total Laryngectomy. Laryngoscope. https://doi.org/10.1002/lary.29857

15. Karkoulias K, Lykouras D, Sampsonas F, Karaivazoglou K, Sargianou M, Drakatos P, Spiropoulos K, Assimakopoulos K (2013) The impact of obstructive sleep apnea syndrome severity on physical performance and mental health. The use of SF-36 questionnaire in sleep apnea. Eur Rev Med Pharmacol Sci 17(4):531-536.

16. Ware J E, Jr., Sherbourne C D (1992) The MOS 36-item short-form health survey (SF-36). I. Conceptual framework and item selection. Med Care 30(6):473-483.

17. Juresa V, Ivankovic D, Vuletic G, Babic-Banaszak A, Srcek I, Mastilica M, Budak A (2000) The Croatian Health Survey-SF-36: I. General quality of life assessment. Coll Antropol 24(1):69-78.

18. Gottlieb D J, Punjabi N M (2020) Diagnosis and Management of Obstructive Sleep Apnea: A Review. JAMA 323(14):1389-1400. https://doi.org/10.1001/jama.2020.3514 
19. Teixeira R C, Cahali M B (2013) Obstructive sleep apnea: is there a difference between vertical and horizontal laryngectomy? Braz J Otorhinolaryngol 79(6):668-672. https://doi.org/10.5935/18088694.20130124

20. Zhou J, Jolly S (2015) Obstructive sleep apnea and fatigue in head and neck cancer patients. Am J Clin Oncol 38(4):411-414. https://doi.org/10.1097/01.coc.0000436086.61460.cb

21. Santana A, Caruso P, Santana P V, Porto G, Kowalski L P, Vartanian J G (2018) Inspiratory muscle weakness, diaphragm immobility and diaphragm atrophy after neck dissection. Eur Arch Otorhinolaryngol 275(5):1227-1234. https://doi.org/10.1007/s00405-018-4923-6

22. Huyett P, Kim S, Johnson J T, Soose R J (2017) Obstructive sleep apnea in the irradiated head and neck cancer patient. Laryngoscope 127(11):2673-2677. https://doi.org/10.1002/lary.26674

23. Tawfik G M, Mostafa E M, Alshareef A, Hmeda A B, Khaled S, Abdelwahed K A, Mahran S A, Agage H S, Amer A E, Emara N S, El-Qushayri A E, Ebied A, Huy N T (2021) Association between radiotherapy and obstructive sleep apnea in head and neck cancer patients: A systematic review and metaanalysis. Auris Nasus Larynx 48(6):1126-1134. https://doi.org/10.1016/j.anl.2021.04.014

24. Rangarajan H, Padmanabhan S, Ranganathan S, Kailasam V (2021) Impact of oral appliance therapy on quality of life (QoL) in patients with obstructive sleep apnea - a systematic review and meta-analysis. Sleep Breath. https://doi.org/10.1007/s11325-021-02483-0

25. Zeineddine S, Rowley J A, Chowdhuri S (2021) Oxygen Therapy in Sleep-Disordered Breathing. Chest 160(2):701-717. https://doi.org/10.1016/j.chest.2021.02.017

26. Inoshita A, Matsumoto F, Ohba S, Sata N, Matsuoka R, Suzuki Y, Ito S, Koiwai H, Shiroshita N, Kasai T, Ikeda K (2021) Severe obstructive sleep apnea after concurrent chemoradiotherapy for laryngeal and hypopharyngeal cancer managed by CPAP. Auris Nasus Larynx. https://doi.org/10.1016/j.anl.2021.05.003

27. Jing Z, Li J, Wang Y, Yuan Y, Zhao D, Hao W, Yu C, Zhou C (2021) Association of smoking status and health-related quality of life: difference among young, middle-aged, and older adults in Shandong, China. Qual Life Res 30(2):521-530. https://doi.org/10.1007/s11136-020-02645-9

28. Beynon R A, Lang S, Schimansky S, Penfold C M, Waylen A, Thomas S J, Pawlita M, Waterboer T, Martin R M, May M, Ness A R (2018) Tobacco smoking and alcohol drinking at diagnosis of head and neck cancer and all-cause mortality: Results from head and neck 5000, a prospective observational cohort of people with head and neck cancer. Int J Cancer 143(5):1114-1127. https://doi.org/10.1002/ijc.31416

\section{Figures}




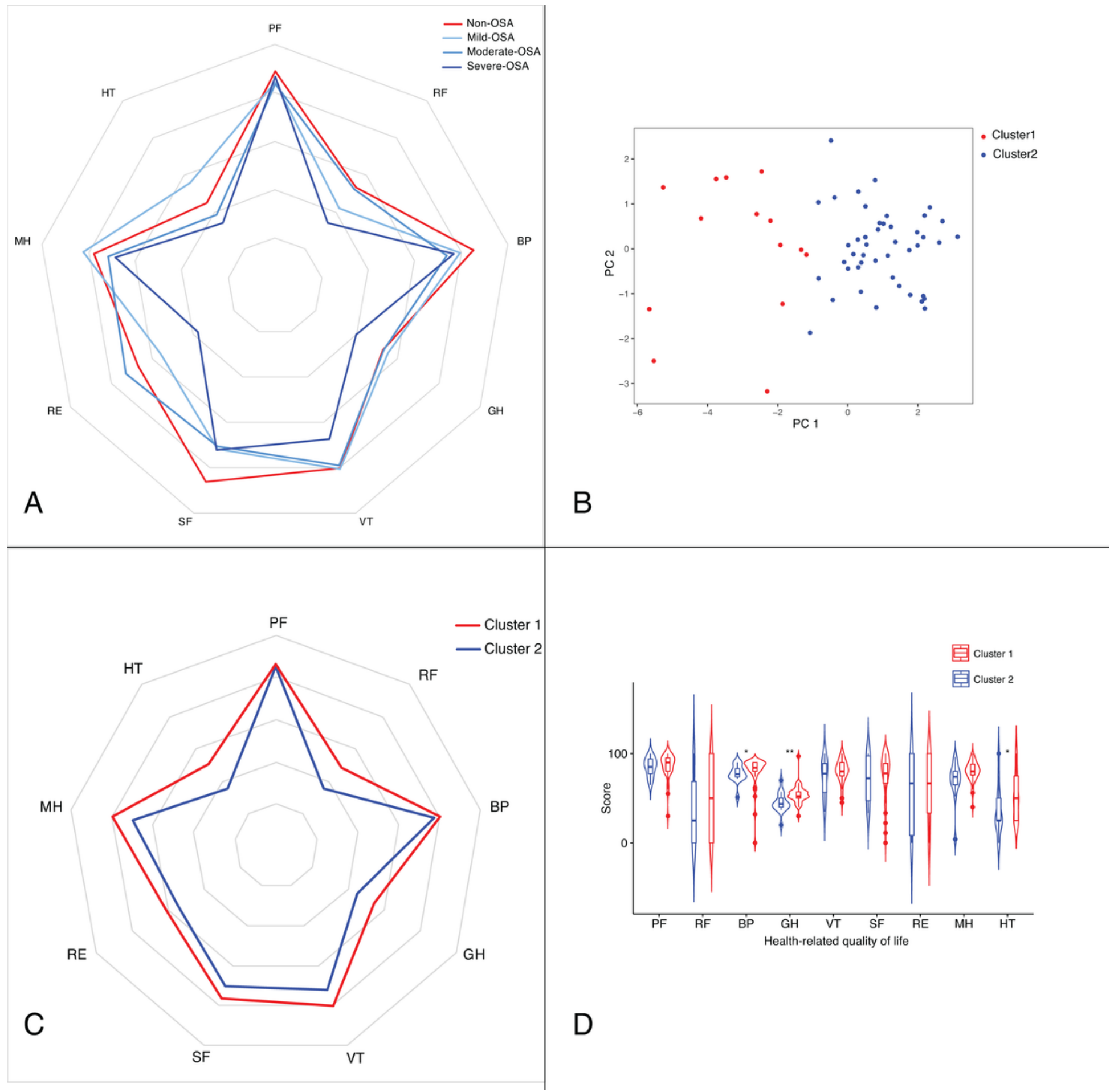

Figure 1

Health-related quality of life assessment in patients with partial laryngectomy for laryngeal cancer. A. Comparisons of SF-36 scores between non-OSA group and OSA subgroups. B. Principal component analysis (PCA)-based K-means clustering. Different colors indicate the two clusters found for the principal components (PC1 and PC2). PC1, labelled "respiratory events", had the greatest loadings on OD and $\mathrm{AHI} ; \mathrm{PC2}$, labelled "oxygen saturation", had the greatest loading on mean $\mathrm{SpO}_{2}$. C. Comparisons of SF-36 scores between cluster 1 and cluster 2. D. Violin plot showing the distribution and level of SF-36 
scores in two clusters. The top and bottom of the embedded box signify the 75th and 25th percentile of the distribution, respectively. The line inside the box represents the median.

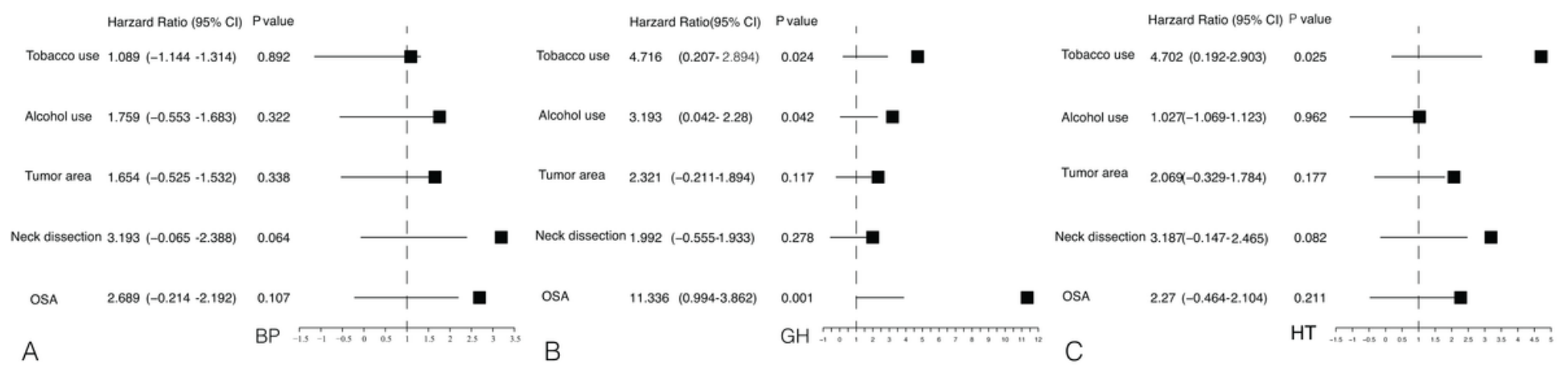

Figure 2

Forest plot of multivariate analysis for factors associated with specific health domains (BP, GH, and $\mathrm{HT}$ ).

\section{Supplementary Files}

This is a list of supplementary files associated with this preprint. Click to download.

- Supplementerymaterial.doc 